\title{
Microstructures of Cu-Ge Alloy Rods Pulled from a Hyperperitectic Melt by the Czochralski Method*1
}

\author{
Yuji Imashimizu and Jirô Watanabé*2 \\ Department of Materials Science and Engineering, Faculty of Engineering and Resource Science, \\ Akita University, Akita 010-8502, Japan
}

$\mathrm{Cu}-\mathrm{Ge}$ alloy rods were pulled from a hyperperitectic melt at three kinds of pulling rates while the melt temperatures were lowered at controlled rates, respectively by the Czochralski method. The relationship between the pulling rate and the Ge concentration in the $\alpha$ phase that solidified first from the melt is approximated by the relation derived from the BPS expression for the effective distribution coefficient. A group of peritectic $\zeta$ grains is formed subsequent to the initial growth of $\alpha$ crystal in the rods pulled at some rates. Growth of the $\zeta$ grains is followed by growth of $\alpha$ phase, resulting in the formation of alternating structures of the primary $\alpha$ and the peritectic $\zeta$ which is accompanied by a simultaneous variation in rod thickness. This appears repeatedly at short intervals along the rod when it is pulled at a high rate. It is concluded that the alternating structures of $\alpha$ phase and peritectic $\zeta$ grains are formed by a peritectic solidification of Ge-rich melt at the cell boundary that develops on a constitutionally supercooled solid-liquid interface, and that their cessation is due to a decrease in Ge concentration of the liquid at the interface, with subsequent growth of the $\zeta$ phase.

(Received December 24, 2002; Accepted August 21, 2003)

Keywords: copper-germanium alloy, hyperperitectic alloy, unidirectional solidification, Czochralski method, effective distribution coefficient, peritectic structure, peritectic solidification, peritectic transformation, constitutional supercooling

\section{Introduction}

The solidification of peritectic alloys involves dendritic growth of the primary $\alpha$ phase and crystallization of the secondary $\beta$ phase from the melt between adjacent $\alpha$ dendrites, ${ }^{1)}$ resulting in the introduction of dendritic structures $^{1-4)}$ in ordinary Bridgman type freezing. Therefore, for the growth of a single phase with peritectic composition it would be necessary to carry out unidirectional solidification under high temperature gradients and low growth rates so as not to grow dendritically. However, it has been shown in the unidirectional solidification of hypoperitectic $\mathrm{Sn}-\mathrm{Sb}$ and $\mathrm{Zn}$ $\mathrm{Cu}$ alloys that the $\alpha$ and $\beta$ phases deposit alternately to form a banded structure under conditions of large $G / R$ ratios ( $G$ and $R$ are the temperature gradient and growth rate, respectively). That is, steady state solidification with planar interface cannot occur. ${ }^{2)}$ Such a banded structure is formed in other alloys which are not only hypoperitectic systems but also hyperperitectic systems. ${ }^{5,6)}$ Thus, it appears generally difficult to prepare peritectic phase crystal using the Bridgman process. However, it may be possible for the $\mathrm{Pb}$ - $\mathrm{Bi}$ system in which a transition from $\alpha$ phase to $\beta$ phase can occur without the banded structure at a very low growth rate with a high temperature gradient, ${ }^{5)}$ resulting in longitudinal separation of the $\alpha$ phase and $\beta$ phase. ${ }^{7)}$ Such a separation into the two phases should be investigated in other peritectic systems.

On the other hand, it has been argued that zone melting of a hyperperitectic alloy rod must divide it into two distinct parts with $\alpha$ and $\beta$ phases. ${ }^{8)}$ This has been verified experimentally in the $\mathrm{CdTe}-\mathrm{In}_{2} \mathrm{Te}_{3}$ system. ${ }^{9)}$ Therefore, the technique may

\footnotetext{
${ }^{* 1}$ This Paper was Partly Presented at the Autumn Meeting of Japan Institute of Metals, held in Sendai, on September 24 to 26, 1997 and at International Conference on Solid-Solid Phase Transformations PTM'99, held in Kyoto Japan, on May 24 to 28, 1999.

${ }^{*}$ Professor Emeritus, Akita University
}

be effective to grow peritectic phase crystal. The Czochralski technique would also be advantageous, since it is possible to control not only the solidification rate but also the melt temperature by the use of a simple apparatus that is commercially available. Moreover, this method enables the unidirectional solidification under high temperature gradients if a thin diameter pulling rod is used. However, the details of how the solidification proceeds during pulling of the peritectic alloy with the Czochralski method has not been extensively investigated.

In this study, $\mathrm{Cu}-\mathrm{Ge}$ alloy was selected as a peritectic system. The copper-rich side of the $\mathrm{Cu}-\mathrm{Ge}$ equilibrium phase $\operatorname{diagram}^{10)}$ is shown in Fig. 1, which indicates that the peritectic reaction,

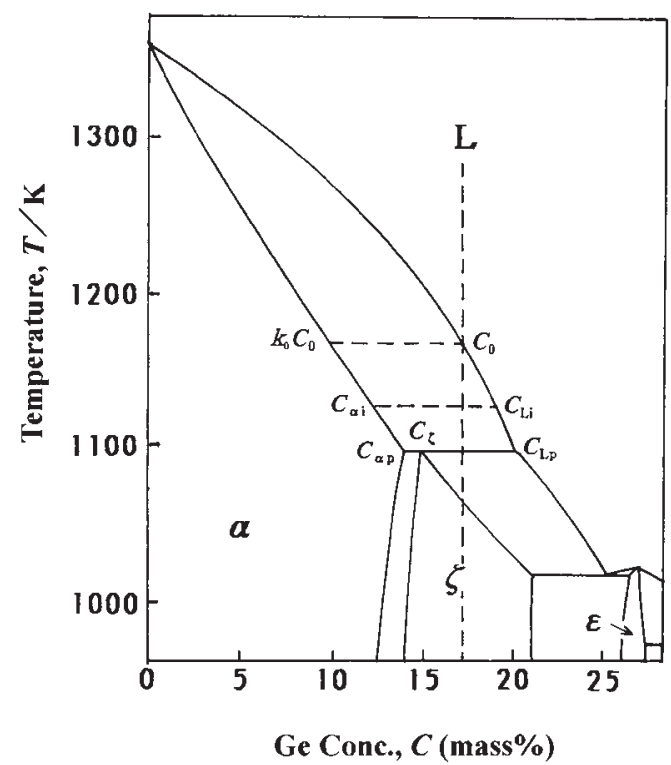

Fig. 1 Copper-rich side of the $\mathrm{Cu}-\mathrm{Ge}$ equilibrium phase diagram. ${ }^{10)}$ 
Solid solution $\alpha(13.5$ mass $\% \mathrm{Ge})+\operatorname{Liquid}(19.5 \operatorname{mass} \% \mathrm{Ge})$

$\rightarrow$ Solid solution $\zeta(14.4$ mass $\% \mathrm{Ge})$

occurs at $1097 \mathrm{~K}$. The growth of the peritectic phase from a hyperperitectic melt is particularly interesting because it can be carried out at low temperatures compared to that from a hypoperitectic melt. Pullings from the hyperperitectic melt containing 17 mass \% germanium were performed under various conditions by the Czochralski method and the microstructures and $\mathrm{Ge}$ distributions in the pulled rod were examined. We will discuss the relationship between the pulling conditions and the features of the peritectic structures to study how the peritectic phase grows from the hyperperitectic melt in the Czochralski process.

\section{Experimental Procedure}

\subsection{Pulling of $\mathrm{Cu}-\mathrm{Ge}$ alloy rods}

99.996 mass\% purity copper (re-electrolyzed copper) and 99.999 mass \% purity germanium were used as starting materials. Pulling of $\mathrm{Cu}-\mathrm{Ge}$ alloy rods was done with a pulling apparatus fitted with the Czochralski head and radio frequency induction heater (DP-150 FB, Kokusai Electric Co. Ltd.). The copper and germanium (total weight about $0.1 \mathrm{~kg}, \mathrm{Cu}-17$ mass $\% \mathrm{Ge}$ ) were melted in a high-purity graphite crucible ( $30 \mathrm{~mm}$ inner diameter) under a stream of purified argon gas. The melt temperature was monitored by a Pt/Pt$\mathrm{Rh}$ thermocouple inserted into the side wall of the crucible and adjusted according to specification by the programmable PID controller. After the melt was held at about $1203 \mathrm{~K}$, a copper seed crystal $5 \mathrm{~mm}$ in diameter and $60 \mathrm{~mm}$ long oriented [111] and tapered to a tip diameter of about $1 \mathrm{~mm}$, was dipped, kept for $600 \mathrm{~s}$ and then pulled upward at a constant rate. The pulling was performed at a low rate of $5.6 \times 10^{-7} \mathrm{~m} / \mathrm{s}$, a medium rate of $1.4 \times 10^{-6} \mathrm{~m} / \mathrm{s}$ or a high rate of $2.8 \times 10^{-6} \mathrm{~m} / \mathrm{s}$, with a rotation rate of $1 \mathrm{rad} / \mathrm{s}$ while the melt temperaure was lowered at a controlled rate. After the melt temperature was lowered at the set rate to about $1153 \mathrm{~K}$, below which the melt starts to solidify, the melt was held at a constant temperature. When the pulling rod attained a length of 30 to $40 \mathrm{~mm}$, it was separated from the melt at a high pulling rate of $4.2 \times 10^{-4} \mathrm{~m} / \mathrm{s}$ and cooled in the furnace. Pulling conditions in this experiment are listed in Table 1.

Table 1 Pulling conditions of $\mathrm{Cu}-\mathrm{Ge}$ alloy rod in this experiment.

\begin{tabular}{cccc}
\hline $\begin{array}{c}\text { Rod } \\
\text { No. }\end{array}$ & $\begin{array}{c}\text { Pulling } \\
\text { rate, }\end{array}$ & \multicolumn{2}{c}{$\begin{array}{c}\text { owering rate of the } \\
\text { melt temperature, }\end{array}$} \\
\cline { 3 - 4 } & $\begin{array}{c}R \\
\left(10^{-6} \mathrm{~m} \mathrm{~s}^{-1}\right)\end{array}$ & $\begin{array}{c}\mathrm{d} T / \mathrm{d} t \\
\left(10^{-3} \mathrm{~K} \mathrm{~s}^{-1}\right)\end{array}$ & $\begin{array}{c}(1 / R) \mathrm{d} T / \mathrm{d} t^{*} \\
\left(10^{3} \mathrm{Km}^{-1}\right)\end{array}$ \\
\hline 1 & 0.56 & 1.7 & 3.0 \\
2 & & 5.6 & 4.0 \\
\hline 3 & 1.4 & 8.3 & 3.0 \\
\hline 5 & 2.8 & & \\
\hline 6 & & & \\
\hline
\end{tabular}

*The rate to a pulling distance.

\subsection{Analysis of microstructures in pulled rods of $\mathrm{Cu}-\mathrm{Ge}$ alloy}

Pulled rods were cut into two or three pieces. Longitudinal sections nearly coincident with the axis of the rods were ground, polished and etched with a 10 percent aqueous solution of $\left(\mathrm{NH}_{4}\right)_{2} \mathrm{~S}_{4} \mathrm{O}_{8}$, and the microstructures examined with an optical microscope. Quantitative compositional analysis of the alloy specimens was performed by EDS (Tracor Northern Co. Ltd). Germanium distributions were investigated by carrying out point analysis at about $0.5 \mathrm{~mm}$ intervals along the entire length of pulled rods as well as at $50 \mu \mathrm{m}$ intervals across some specified microstructures.

\section{Experimental Results}

\subsection{External shapes of $\mathrm{Cu}-\mathrm{Ge}$ alloy rods and $\mathrm{Ge}$ distribution along the rod length}

Examples of external shapes of pulled $\mathrm{Cu}-\mathrm{Ge}$ alloy rods and macroscopic distributions of germanium along the rod length are shown in Fig. 2. In rod No. 1, which was pulled at the low rate, the diameter was nearly constant in the initial half length, but increased gradually toward the end (Fig. 2(a)). The Ge concentration was nearly constant in most of the rod except for the thick end, in which it increased sharply. Although rod No. 2 was pulled at the same rate as No. 1, the former was thicker and had a higher Ge concentration than the latter. The shape was rather similar to that of rods No. 3 and 4 , which were pulled at the medium rate. The thickness of rods No. 3 and 4 did not appreciably increase in the initial portion, but increased and then decreased in the middle portion, showing a variation that tended to repeat itself in the second half (Fig. 2(b)). In rods No. 5 and 6, which were pulled at the high rate, the increase and decrease in thickness were alternately repeated at short intervals along the rod length (Fig. 2(c)). In the rods pulled at medium and high rates, the Ge concentration generally tended to change along the rod length, in step with the thickness, so that it was at a maximum near the thicker portions with the peritectic composition of 14.4 mass $\%$, but it also increased steeply to a high value at the terminal end.

The average Ge concentrations in the initial $15 \mathrm{~mm}$ of pulled rods are given in Table 2. The first phase that solidified from the melt of $\mathrm{Cu}-17$ mass\% Ge alloy was the $\alpha$ phase containing 10 to 12 mass $\% \mathrm{Ge}$, of which the highest concen-

Table 2 The average Ge concentration in the initial $15 \mathrm{~mm}$ (primary $\alpha$ phase) of pulled rods, and the distance from the seed to the first formed $\zeta$ structure.

\begin{tabular}{ccc}
\hline $\begin{array}{c}\text { Rod } \\
\text { No. }\end{array}$ & $\begin{array}{c}\text { Ge concentration } \\
\text { of the primary } \alpha, \\
C(\operatorname{mass} \%)\end{array}$ & $\begin{array}{c}\text { Location of the } \\
\text { first formed } \zeta, \\
*^{*} / \mathrm{mm}\end{array}$ \\
\hline 1 & 10.6 & 25 \\
2 & 10.9 & 20 \\
\hline 3 & 10.4 & 15 \\
4 & 10.7 & 17 \\
\hline 5 & $11.9^{* *}$ & 1.6 \\
6 & $11.9^{* *}$ & 4.4 \\
\hline
\end{tabular}

* Distance from the seed.

** Region except for the secondary phase. 
(a)

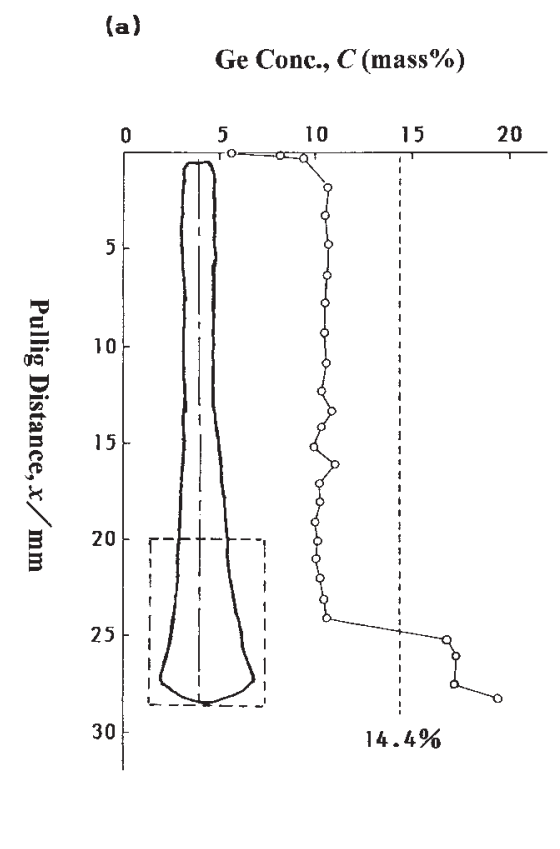

(b) Ge Conc., $C$ (mass\%) (c)

Ge Conc., $C$ (mass\%)
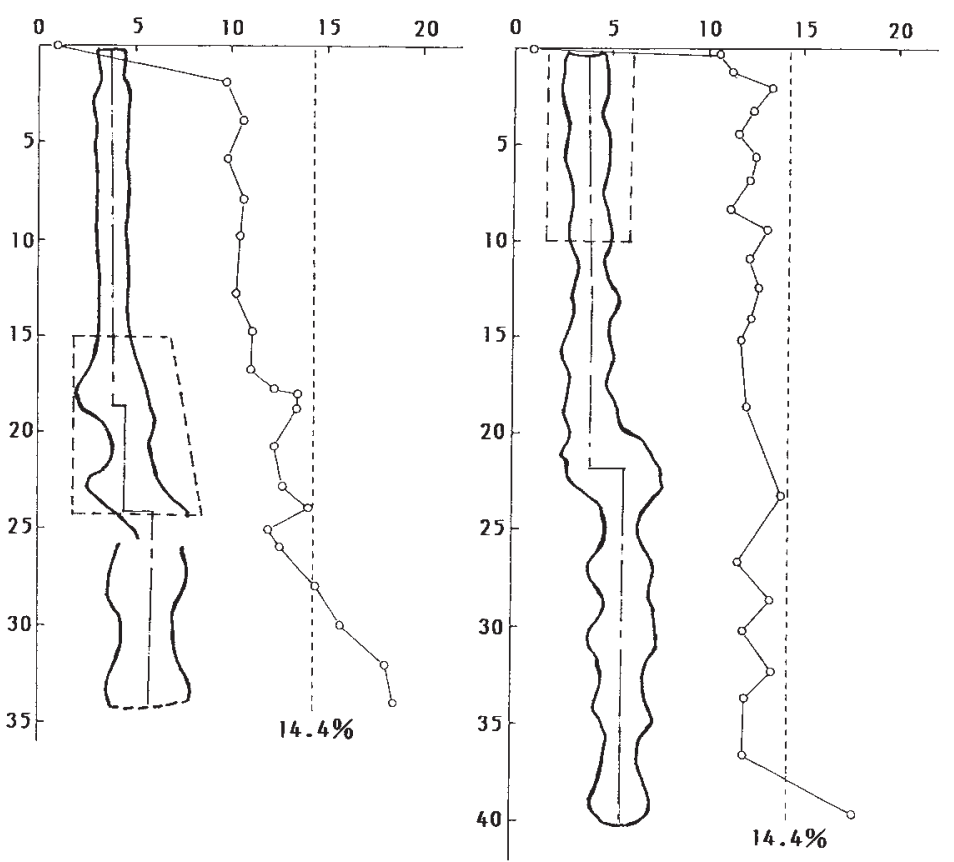

Fig. 2 External shapes and the Ge distributions along the length of $\mathrm{Cu}-\mathrm{Ge}$ alloy rods pulled at (a) low, (b) medium and (c) high rates.
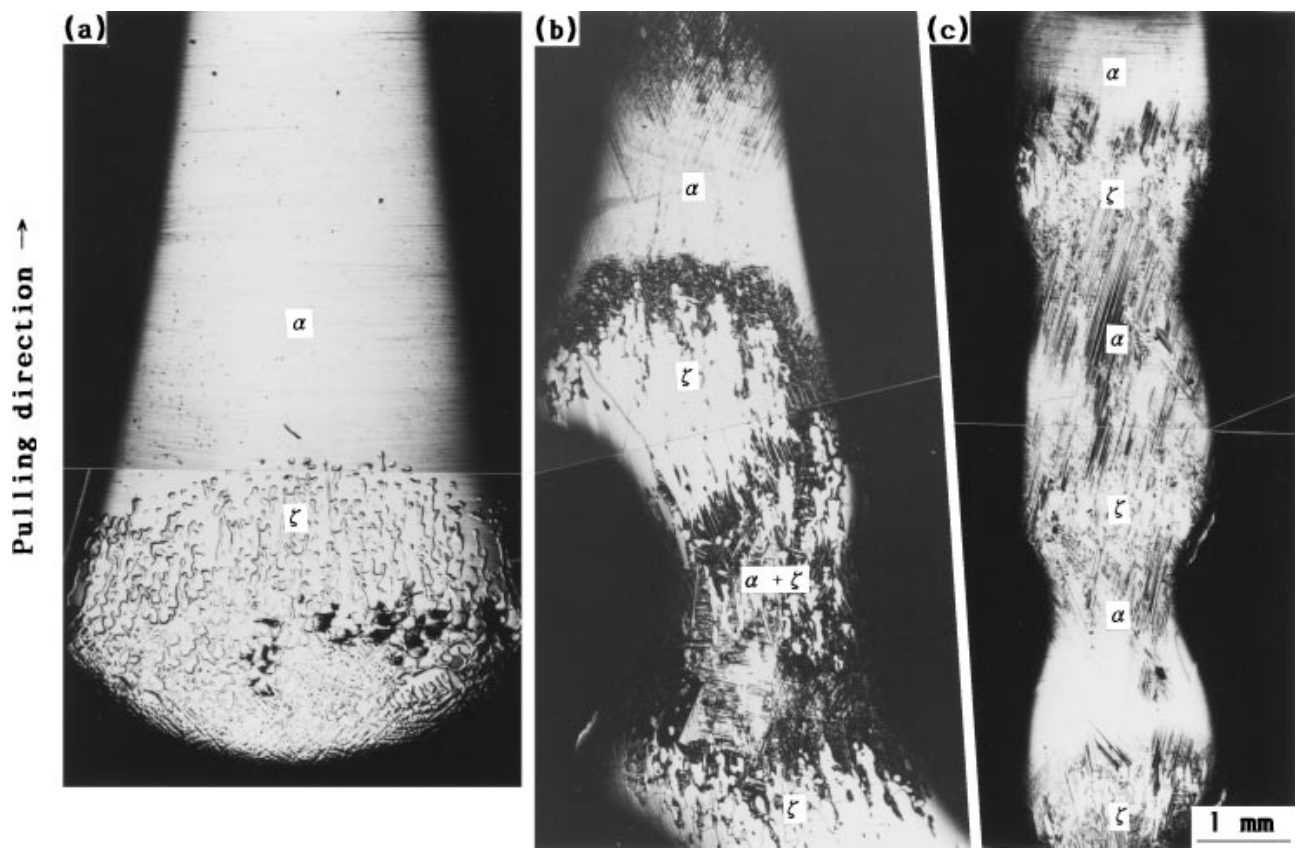

Fig. 3 Photographs of the microstructures developed on longitudinal sections of $\mathrm{Cu}-\mathrm{Ge}$ alloy rods pulled at (a) low, (b) medium and (c) high rates, which cover the areas surrounded by the broken line in Figs. 2(a), (b) and (c), respectively.

tration appeared in the rods pulled at the high rate.

\subsection{Microstructures and Ge distribution}

Figures 3(a), (b) and (c) show low-magnification micrographs of longitudinal sections of the rods pulled at low, medium and high rates, which cover the areas surrounded by broken lines in Figs. 2(a), (b) and (c), respectively. A comparison of Fig. 2 with Fig. 3 shows that the first-formed $\alpha$ phase was a single crystal in all rods because no grain boundaries appeared, and the traces of Widmanstätten $\zeta$ lamellae which should be formed martensitically along the octahedral habit planes of supersaturated $\alpha(\mathrm{fcc})^{11)}$ aligned along the same directions. On the other hand, the characteristic microstructures of secondary phases were first formed in the thicker portions of the rods, which were located at different distances from the seed depending on the pulling 

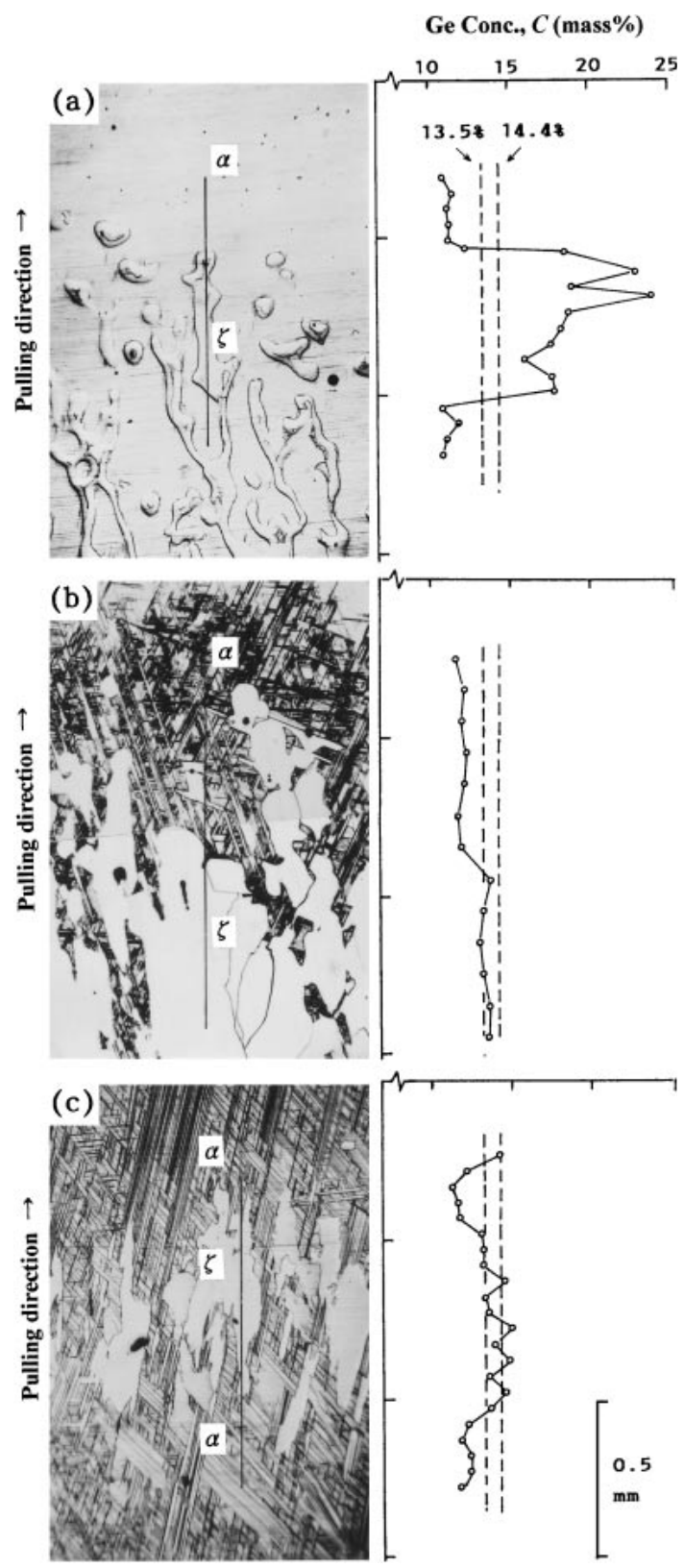

Fig. 4 Magnified views of the microstructures shown in Fig. 3 and the microscopic Ge distributions along the lines drawn on the micrographs, which were formed in rods pulled at (a) low, (b) medium and (c) high rates.

rate, as shown in Table 2. Figure 4 shows optical micrographs of a part of the microstructures shown in Fig. 3, and the Ge distributions along the line drawn on the micrographs, taken at a higher magnification. The average Ge concentrations over a growth distance crossing the $\alpha$ matrix and the secondary phase are shown in Table 3.

In rod No. 1, which was pulled at the low rate, an islandlike structure was introduced in the last thick portion (Figs. 3(a) and 4(a)). The Ge concentration in the island is higher than the peritectic composition $C_{\zeta}$, while that in the $\alpha$ matrix is low compared with composition $C_{\alpha \mathrm{p}}$ giving rise to peritectic reaction (Fig. 4(a), Table 2). In rod No. 2, a mixed structure of Widmannstätten lamellae and round grains of secondary phase were formed in a thicker portion of the second half.
Table 3 The Ge concentration of the secondary $\zeta$ grains and their neighboring matrices.

\begin{tabular}{ccc}
\hline \multirow{2}{*}{$\begin{array}{c}\text { Rod } \\
\text { No. }\end{array}$} & \multicolumn{2}{c}{$\begin{array}{c}\text { Ge concentration, } \\
C(\text { mass } \%)\end{array}$} \\
\cline { 2 - 3 } & $\begin{array}{c}\text { Matrix } \\
\alpha \text { phase }\end{array}$ & $\begin{array}{c}\text { Secondary } \\
\zeta \text { phase }\end{array}$ \\
\hline 1 & $11.6^{*}$ & $18.1^{*}$ \\
2 & $12.6^{* *}$ & $14.8^{* *}$ \\
\hline 3 & 12.0 & 13.7 \\
4 & 13.0 & 14.5 \\
\hline 5 & 12.1 & 13.4 \\
6 & 12.3 & 14.0 \\
\hline
\end{tabular}

* For the structure shown in Fig. 4(a).

** For a structure similar to that formed in the rods pulled at higher rates.

In rods No. 3 and 4, which were pulled at the medium rate, the initial growth of $\alpha$ crystal was followed by a succession of micromorphologies in the middle portion: $\alpha$ phase containing prominently developed Widmanstätten lamellae, a group of secondary phase grains, and $\alpha$ phase, as shown in Fig. 3(b). The Ge concentration of the secondary $\zeta$ grains approximately coincides with $C_{\zeta}$ (Fig. 4(b) and Table 3). The formation of the $\zeta$ grains was accompanied by a decrease in thickness, and subsequently $\alpha$ phase instead of $\zeta$ grains, which still contained a few dispersed $\zeta$ grains, grew again. It was accompanied with an increase in thickness and the second group of $\zeta$ grains introduced alternatively.

In rods No. 5 and 6 , which were pulled at the high rate, after $\alpha$ phase containing dense Widmanstätten lamellae grew initially, a group of $\zeta$ grains, the composition of which is nearly equal to $C_{\zeta}$, appeared in the initial thicker portion (Figs. 3(c) and 4(c)). It is noticeable that the thickness of the rods decreased with growth of the $\zeta$ grains, but increased again with the subsequent growth of $\alpha$ phase. The alternating structures with a variation in thickness repeatedly formed at short intervals along the rod length. Figure 5(a) shows a later group of $\zeta$ grains formed in the second half of the rods. It can be seen that the $\zeta$ grains became large compared to those in the initial portion.
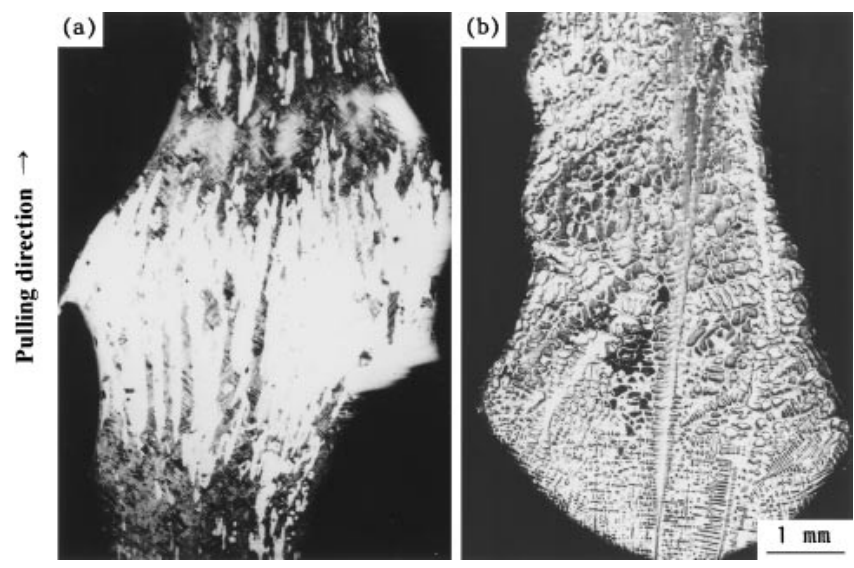

Fig. 5 Low-magnification photographs of the microstructures developed on longitudinal sections of the (a) middle and (b) last portions of the rod pulled at a high rate. 
The terminal end of rods pulled at the medium and high rates generally exhibited a complicated structure, which contained dendritic structure as shown in Fig. 5(b) and had a very high Ge concentration compared to $C_{\zeta}$ (Figs. 2(b) and (c)).

\section{Discussion}

\subsection{Relationship between Ge concentration in pulled rod and its pulling rate}

When a rod-like alloy crystal is grown unidirectionally from the stirred melt with an initial composition $C_{0}$ by the Czochralski method, the solute concentration in the solid phase at the solid-liquid interface $C_{\mathrm{S}}$ is given by

$$
C_{\mathrm{S}}(f)=k C_{0}(1-f)^{k-1},
$$

where $f$ is the fraction of the melt solidified and $k$ the effective distribution coefficient, and $C_{0}(1-f)^{k-1}$ corresponds to the bulk concentration in the melt $C_{\mathrm{Lb}}(f)$. For a CuGe alloy with an equilibrium distribution coefficient $k_{0}$ less than unity, the solute concentration in the liquid at the solidliquid interface $C_{\mathrm{Li}}(f)$ becomes higher than $C_{\mathrm{Lb}}(f)$ because of the rejection of solute atoms from the advancing interface. Hence the Ge distribution profile near the advancing solidliquid interface can be schematically drawn as shown in Fig. 6. In this figure, the growth distance $x$ is related to $f$ as $x=4 f W /\left(\rho \pi d^{2}\right)$, where $W$ is the initial weight of the melt, $\rho$ the density of the melt and $d$ the diameter of the solidifying rod.

Provided that equilibrium prevails at the interface virtually independently of growth rate, Ge concentration in the $\alpha$ solid phase at the solid-liquid interface $C_{\alpha \mathrm{i}}(x)$ can be related to that in the liquid phase $C_{\mathrm{Li}}(x)$ with the equilibrium distribution coefficient $k_{0}$ as

\section{Ge Concentration}

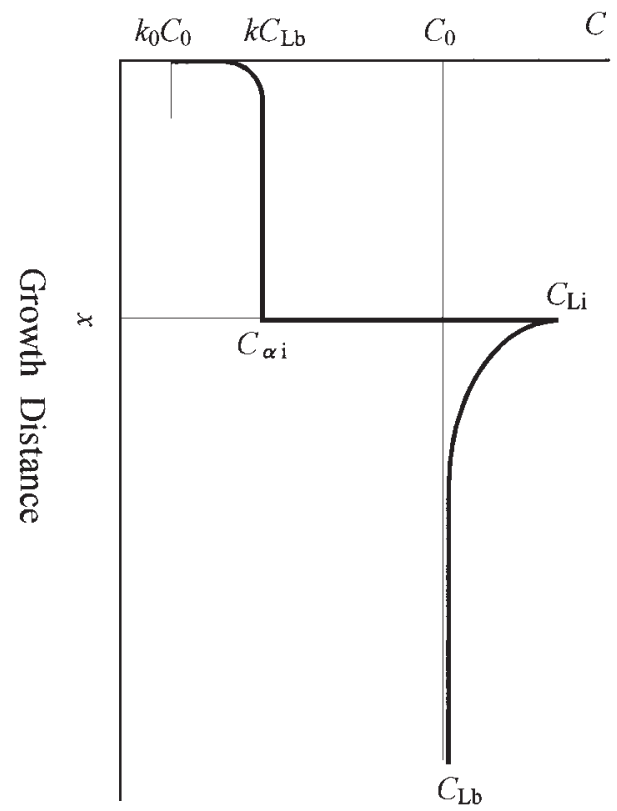

Fig. 6 Schematic drawing of the Ge distribution in the neighborhood of the advancing solid-liquid interface when $\mathrm{Cu}-\mathrm{Ge}$ alloy solidifies unidirectionally from the stirred melt.

$$
C_{\alpha \mathrm{i}}(x)=k_{0} C_{\mathrm{Li}}(x)
$$

Moreover, according to the BPS theorem, ${ }^{12,13)}$ if the growth rate of the solid phase is assumed to be nearly equal to the pulling rate $R, k$ is expressed as

$$
k=\frac{k_{0}}{k_{0}+\left(1-k_{0}\right) \exp (-R \delta / D)}
$$

where $D$ is the diffusion coefficient of solute atoms in the liquid and $\delta$ the thickness of the diffusion layer at the interface. $^{12)} \delta$ is given by

$$
\delta=1.6 D^{1 / 3} v^{1 / 6} \omega^{-1 / 2},
$$

where $v$ is the kinematic viscosity and $\omega$ is the angular velocity of the solid. ${ }^{12)}$

Since the solidified fraction in the present pulling of $\mathrm{Cu}-\mathrm{Ge}$ alloy is estimated to be in the range of $f \leq$ $\rho \pi d^{2} x_{\max } / 4 W=0.01$, assuming $d=2 \mathrm{~mm}, x_{\max }=40 \mathrm{~mm}$, $W=0.1 \mathrm{~kg}$ and $\rho=8 \times 10^{3} \mathrm{~kg} / \mathrm{m}^{3}$, eq. (2) can be approximately expressed as

$$
C_{\alpha \mathrm{i}}(x) \approx k C_{0} .
$$

That is, the Ge concentration in the pulling rod, especially in the initial portion, is almost independent of the pulled length. This is supported by the Ge concentration profiles of initial halves of the pulled rods shown in Fig. 2. Figure 7 shows the plots of Ge concentration in the rods pulled from the melt of $\mathrm{Cu}-17$ mass \% Ge as a function of pulling rate. Open circles designate the measured values shown in Table 2 and the solid line is a curve calculated from eqs. (4) and (6) assuming a proper value $\left(1.96 \times 10^{5} \mathrm{~s} / \mathrm{m}\right)$ for $\delta / D$. The measured values generally agree with the calculated curve and the value assumed for $\delta / D$ is valid because it is estimated to be $0.9 \times 10^{5} \mathrm{~s} / \mathrm{m}$ from eq. (5) since $\omega$ is $1 \mathrm{rad} / \mathrm{s}$, if we assume $D=2.0 \times 10^{-9} \mathrm{~m}^{2} / \mathrm{s}$ (an estimated value of the diffusion coefficient of germanium in the copper alloy melt at $1100 \mathrm{~K}$ ) and $v=5 \times 10^{-7} \mathrm{~m}^{2} / \mathrm{s}$ (a value for the copper melt at melting point) referring to a data book. ${ }^{14)}$ This shows that the

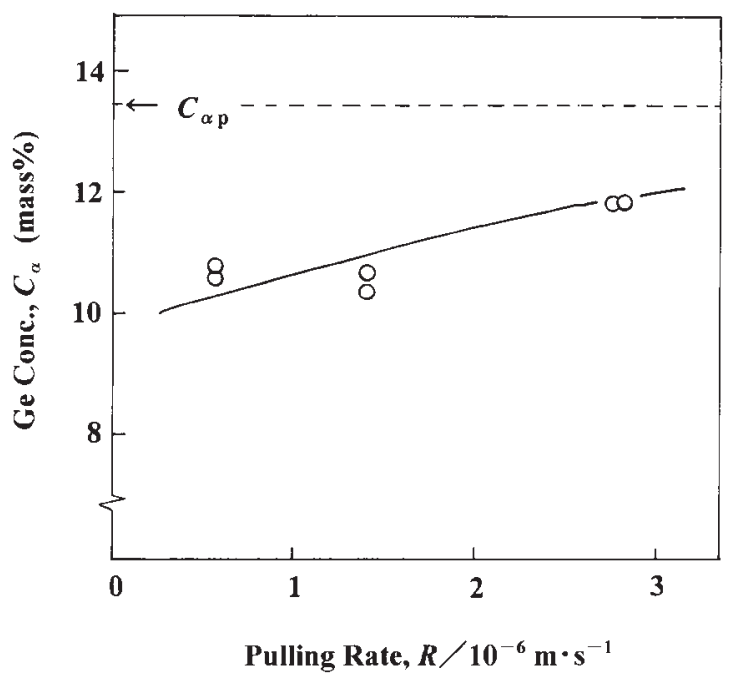

Fig. 7 Plot of the Ge concentration in the initial $15 \mathrm{~mm}$ (primary $\alpha$ phase) as a function of pulling rate. Open circles indicate the measured values shown in Table 2 and the solid line is a calculated curve. 
relationship between the Ge concentration of the growing $\alpha$ solid phase and the pulling rate is approximated by the relation derived from the BPS expression for the effective distribution coefficient, though it should be applied to the dilute alloy. ${ }^{13)}$

\subsection{Relationship between peritectic structure and pull- ing rate}

\subsubsection{Influence of lowering of the melt temperature}

In crystal pulling, the temperature gradient $G_{\mathrm{L}}$ in the liquid at the solid-liquid interface is assumed to be proportional to the difference $\Delta T$ between the melt temperature $T$ just below the liquid element lifted by the pulling rod and the interface temperature $T_{\mathrm{i}}$, according to the theory ${ }^{15)}$ concerning the cross section of Czochralski crystals. In this experiment, the temperature of the bulk melt $\left(T_{\mathrm{b}}\right)$ was lowered at a rate that depended on the pulling rate (Table 1 ). Thus, $T$ was lowered at a corresponding rate. On the other hand, when $\mathrm{Cu}-\mathrm{Ge}$ alloy rod is pulled at a constant rate $R, T_{\mathrm{i}}$ is surmised to correspond to the melting temperature of the $\alpha$ phase of composition $C_{\alpha \mathrm{i}}(x)$ given by eqs. (4) and (6). (Figs. 1 and 6). Hence, lowering of $T_{\mathrm{b}}$ decreases $\Delta T=T-T_{\mathrm{i}}$ and consequently $G_{\mathrm{L}}$, resulting in an increase in the diameter of the rod, as predicted from the theory. ${ }^{15)}$ Thus, the increase in thickness of the pulled rod shown in Figs. 2 and 3 is thought to be attributable to a decrease in the temperature gradient $G_{\mathrm{L}}$ at the interface.

On the other hand, it is known that the advancing solidliquid interface becomes unstable to form a cellular structure due to constitutional supercooling under temperature gradients lower than a critical value. ${ }^{16-18)}$ The critical temperature gradient increases with an increase in growth rate. Hence the constitutional supercooling would arise at an earlier stage under the higher pulling rate, if the melt temperature lowering rates at a particular pulling distance were the same between different pullings. This applies to the present experiment because the ratio of the lowering rate of the melt temperature to the pulling rate, $(1 / R)(\mathrm{d} T / \mathrm{d} t)$ was about the same between the pullings at different rates as shown in Table 1. Therefore, the variations of thickness and microstructure in the pulled rods can be explained as follows.

\subsubsection{Formation of the secondary $\zeta$ grains}

The formation of an island-like structure with a high Ge concentration in the last thick portion of rod No. 1 (Figs. 3(a) and 4(a)) can be attributed to a constitutional supercooling that should be brought about latest in this case because of the low pulling rate. The structure is thought to arise as a solidification of Ge-rich melt penetrates into the solid at the cell boundaries and nodes developed on the supercooled solid-liquid interface. ${ }^{17)}$

On the other hand, constitutional supercooling should arise at an earlier stage in the pulling of rods No. 3 and 4 at the medium rate. Hence the group of $\zeta$ grains in the thickened middle portion of the rods (Fig. 3(b)) is also thought to be formed via a solidification of the Ge-rich melt trapped at cell boundaries developed on the supercooled interface. However, those $\zeta$ grains were formed in contact with each other (Fig. 4(b)), differing from the above island-like structures shown in Fig. 4(a) This shows that the $\alpha$ phase between cell boundaries transformed into the $\zeta$ phase at the same time that
Ge-rich melt penetrating into cell boundaries solidified to form the $\zeta$ phase. The Ge concentration of the $\zeta$ grains is nearly equal to the peritectic composition $C_{\zeta}$ (Fig. 4(b) and Table 3 ). Thus, the $\zeta$ grains are considered to be the peritectic phase which was formed by a peritectic reaction including $\alpha \rightarrow \zeta$ peritectic transformation ${ }^{19,20)}$ that nucleates at the $\alpha /$ Ge-rich melt interface, as described in the previous paper. ${ }^{21)}$ From here on we will refer to the formation of the $\zeta$ grains as peritectic solidification. A mixed structure of the $\alpha$ matrix and a dispersed round $\zeta$ grains of composition $C_{\zeta}$ (Table 3) was formed in rod No. 2. This is attributed to the peritectic solidification caused by a fluctuation in growth conditions.

With high-rate pulling, constitutional supercooling should arise at the earliest stage of pulling. This resulted in the formation of $\zeta$ grains of composition $C_{\zeta}$ in the initial portion of the pulled rods (Fig. 4(c) and Table 3) by the same process as that in the medium-rate pulling. However, the features of the microstructure of $\zeta$ grains were different from that in the rods pulled at the medium rate. The reason for this is that when pulling proceeded at the high rate, the composition of $\alpha$ phase $C_{\alpha \mathrm{i}}(x)$ was nearer to $C_{\alpha \mathrm{p}}$ than when pulling was done at the medium rate as shown in Fig. 7, and that the $\zeta$ grains were formed via the peritectic solidification under a smaller degree of constitutional supercooling.

\subsubsection{Formation of alternating structures of the primary $\alpha$ and the peritectic $\zeta$}

In the rods pulled at the medium and high rates, a group of peritectic $\zeta$ grains was formed subsequent to the growth of $\alpha$ phase containing prominently developed Widmanstätten lamellae, but the growth of $\zeta$ grains was followed by growth of $\alpha$ phase, resulting in the formation of alternating structures of $\alpha$ and peritectic $\zeta$. This was accompanied by a simultaneous variation in rod thickness (Figs. 3(b) and (c)). These things are interpreted by referring to the schematic drawings in Figs. 8 and 9, as follows.

The development of a cellular substructure of many depressions ${ }^{16)}$ formed from Ge-rich melt on the constitutionally supercooled solid-liquid interface (Fig. 8) is thought to increase the average Ge concentration in the liquid along the interface and consequently that in the $\alpha$ solid. We assume that the average $\mathrm{Ge}$ concentrations increase from $C_{\mathrm{Li}}$ and $C_{\alpha \mathrm{i}}$ (on the first thick line in Fig. 9) to about the limits of the peritectic horizontal $C_{\mathrm{Lp}}$ and $C_{\alpha \mathrm{p}}$ (on the first thin line in Fig. 9), respectively. Although it is not known whether those increased average concentrations coincide with $C_{\mathrm{Lp}}$ and $C_{\alpha \mathrm{p}}$, respectively, the assumption seems reasonable qualitatively.

We surmise that such an increase in $C_{\alpha \mathrm{i}}$ resulted in the growth of $\alpha$ phase containing Widmannstätten lamellae that precipitated during cooling and the subsequent formation of peritectic $\zeta$ grains by the peritectic solidification (Fig. 8(b)). The increase in the average Ge concentrations in the liquid and solid along the interface also lowered the interface temperature $T_{\mathrm{i}}$ (Fig. 1) and consequently increased the temperature gradient $G_{\mathrm{L}}$, which is deduced from the discussion in 4.2.1. This produced a decrease in rod thickness, with the formation of a group of $\zeta$ grains.

However, the subsequent growth of the $\zeta$ grains (Fig. 8(c)) with a higher Ge concentration than $\alpha$ phase is thought to decrease the number of $\mathrm{Ge}$ atoms rejected from the advancing interface. This resulted in a decrease in the average Ge 
(a)

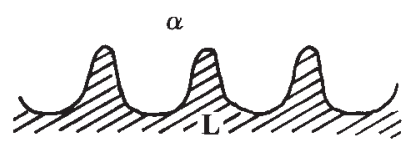

(b)

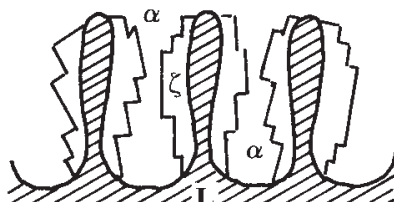

(c)

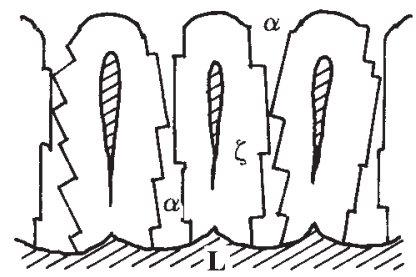

(d)

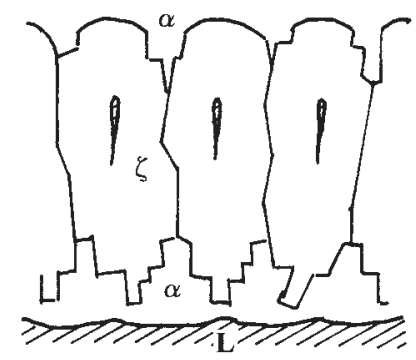

Fig. 8 Illustration for the formation process of a group of peritectic $\zeta$ grains. (a) the solid-liquid interface at the onset of constitutional supercooling, (b) peritectic solidification, (c) growth of $\zeta$ grains, and (d) cessation of $\zeta$ grain growth and growth of the second $\alpha$ phase.

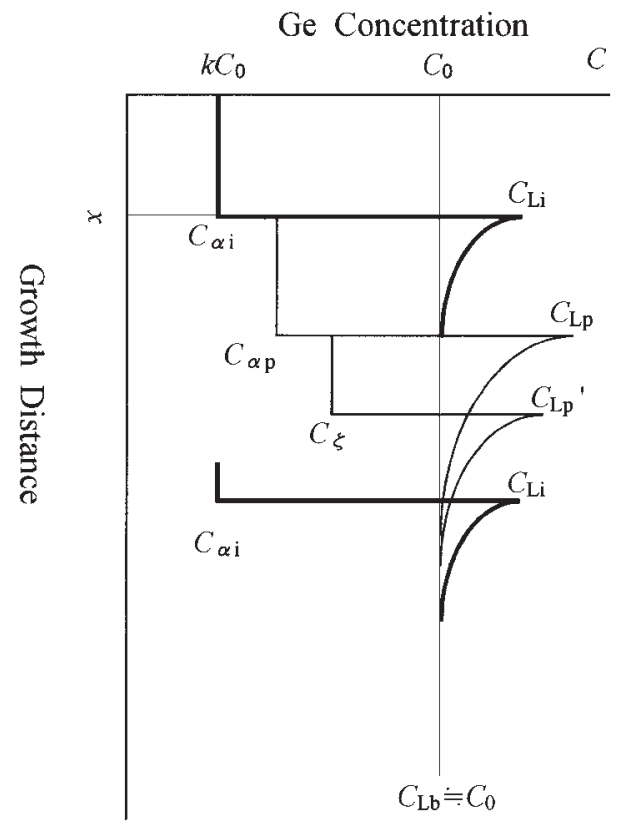

Fig. 9 Schematic diagram of the presumed change of the Ge distribution near the solid-liquid interface that explains the formation of alternate $\alpha$ and $\zeta$ phases. The thick and thin lines show the distribution for growing with the planar interface and that with the cellular interface under a condition of constitutional supercooling, respectively. concentration in the liquid at the interface from $C_{\mathrm{Lp}}$ to $C_{\mathrm{Lp}}{ }^{\prime}$, and even further to $C_{\mathrm{Li}}$ (on the second thin and thick lines in Fig. 9), with cancellation of an increase in Ge concentration due to constitutional supercooling. Such a variation in the liquid composition at the interface allowed $\alpha$ phase to grow again, instead of $\zeta$ phase (Fig. 8(d)), and increased $T_{\mathrm{i}}$. The increase in $T_{\mathrm{i}}$ decreased the temperature gradient $G_{\mathrm{L}}$ in further pulling and gradually increased the rod thickness again, This destabilized the advancing interface and introduced the second group of $\zeta$ grains (Fig. 3(b) and (c)).

The $\zeta$ grains in the initial portion of the rods that were pulled at the high rate were smaller than those in the middle portion of the rods that were pulled at the medium rate (Figs. 4(b) and (c)), but the later group of $\zeta$ grains in the second half of the former became large to form a morphology similar to that in the latter (Fig. 5(a)). This is attributed to an increase in the magnitude of constitutional supercooling with lowering of the melt temperature.

\subsubsection{Formation of related structures}

In rod No. 1, island-like structures with a higher $\mathrm{Ge}$ concentration than $C_{\zeta}$ (Fig. 4(a)) which are different from peritectic products were formed via a solidifying of the Gerich melt at the cellular interface and few Widmannstätten lamellae precipitated in the $\alpha$ phase. The reason for this is that the $C_{\alpha \mathrm{i}}$ was low compared to $C_{\alpha \mathrm{p}}$ even at the onset of constitutional supercooling because of low pulling rate (Table 3). On the other hand, the microstructures in the terminal end of the rods pulled at the medium and high rates (Fig. 5(b)) were very complicated and contained much more germanium than in the peritectic $\zeta$ phase (Figs. 2(b) and (c)). This shows that those structures are also not peritectic products, but the $\zeta$ phases or a probable eutectic of $\zeta$ and $\varepsilon$ phases (see Fig. 1) that solidified directly from a Ge-rich melt. The formation of such structures is attributed to dendritic growth that should proceed under the large degree of constitutional supercooling that arose from a higher pulling rate.

\section{Conclusions}

$\mathrm{Cu}-\mathrm{Ge}$ alloy rods were pulled from a hyperperitectic melt at three kinds of pulling rates while the melt temperatures were lowered at controlled rates, respectively by the Czochralski method. The microstructures and the Ge distributions in the pulled rods were examined.

(1) The relationship between the pulling rate and the Ge concentration of the $\alpha$ phase that solidified first from the melt is approximated by the relation derived from the BPS expression for the effective distribution coefficient.

(2) A group of peritectic $\zeta$ grains is formed subsequent to the initial growth of $\alpha$ crystal in the rods pulled at some rates. The growth of $\zeta$ phase is followed by $\alpha$ phase growth, producing alternating structures of the primary $\alpha$ and the peritectic $\zeta$ which is accompanied by a simultaneous variation in rod thickness. This appears repeatedly at short intervals along the rod when it is pulled at a high rate.

(3) The alternating structures are formed by a peritectic solidification of Ge-rich melt at the cell boundary that develops on a constitutionally supercooled solid-liquid interface, and their cessation is due to a decrease in Ge 
concentration in the liquid at the interface, with subsequent growth of the $\zeta$ phase.

\section{Acknowledgments}

The authors wish to express their thanks to Mr. Mazlan Mohammad, who was a graduate student at Akita University, for his kind help with the experiments.

\section{REFERENCES}

1) D. R. Uhlmann and G. A. Chadwick: Acta Metall. 9 (1961) 835-840.

2) A. P. Titchener and J. A. Spittle: Acta Metall. 23 (1975) 497-502.

3) H. Fredriksson and T.Nylén: Metal Sci. 16 (1982) 283-294.

4) D. H. St. John and L. M. Hogan: J. Mater. Sci. 19 (1984) 939-948.

5) K. Tokieda, H. Yasuda, I. Ohnaka: Mater. Sci. Eng. A262 (1999) 238245.

6) H. Yasuda, N. Notake, K. Tokieda, I. Ohnaka: J. Crys. Growth 210 (2000) 637-645.

7) R. Trivedi: Metall. Mater. Trans. A 26A (1995) 1583-1589.

8) C. H. L. Goodman: Research, (London) 7 (1954) 168-177.
9) D. R. Mason and J. S. Cook: J. Appl. Phys. 32 (1961) 475-477.

10) R. W. Olesinski and G. J. Abbaschian, in: Binary Alloy Phase Diagrams, 1, (ASM, Ohio, 1986) pp. 919-921.

11) P. S. Kotval and R. W. K. Honeycombe: Acta Metall. 16 (1968) 597607.

12) J. A. Burton, R. C. Prim and W. P. Slichter: J. Chem. Phys. 21 (1953) 1987-1991.

13) J. A. Burton, E. D. Kolb, W. P. Slichter and J. D. Struthers: J. Chem. Phys. 21 (1953) 1991-1996.

14) Nippon Kinzoku Gakkai: Kinzoku Dêta Bukku, (Maruzen, Tokyo, $1993)$ pp. 16, 57.

15) R. G. Pohl: J. Appl. Phys. 25 (1954) 668-669.

16) H. Biloni, G. F. Bolling and G. S. Cole: Trans. Metall. Soc. AIME 236 (1966) 930-935.

17) R. M. Sharp and A. Hellawell: J. Crys. Growth 11 (1971) 77-91.

18) D. T. J. Hurle: Solid-State Electron. 3 (1961) 37-44.

19) M. Hillert, in: Solidification and Casting of Metals, (The Metals Society, London, 1979) pp. 81-87.

20) K. Matsuura and M. Kudoh: Mater. Trans., JIM 39 (1998) 203-210.

21) Y. Imashimizu, J. Watanabé and M. Mohammad: Proc. Int. Conf. on Solid-Solid Phase Transformations '99 (JIMIC-3), (The Japan Inst. Metals, 1999) pp. 273-276. 